\title{
Understanding the impact of genetic testing for inherited retinal dystrophy
}

\author{
Ryan Combs ${ }^{1}$, Marion McAllister ${ }^{2}$, Katherine Payne ${ }^{1}$, Jo Lowndes ${ }^{3}$, Sophie Devery ${ }^{4}$, Andrew R Webster ${ }^{4,5}$, \\ Susan M Downes ${ }^{3}$, Anthony T Moore ${ }^{4,5}$, Simon Ramsden ${ }^{6}$, Graeme Black ${ }^{1,6}$ and Georgina Hall ${ }^{\star, 6}$
}

The capability of genetic technologies is expanding rapidly in the field of inherited eye disease. New genetic testing approaches will deliver a step change in the ability to diagnose and extend the possibility of targeted treatments. However, evidence is lacking about the benefits of genetic testing to support service planning. Here, we report qualitative data about retinal dystrophy families' experiences of genetic testing in United Kingdom. The data were part of a wider study examining genetic eye service provision. Twenty interviewees from families in which a causative mutation had been identified by a genetic eye clinic were recruited to the study. Fourteen interviewees had chosen to have a genetic test and five had not; one was uncertain. In-depth telephone interviews were conducted allowing a thorough exploration of interviewees' views and experiences of the benefits of genetic counselling and testing. Transcripts were analysed using thematic analysis. Both affected and unaffected interviewees expressed mainly positive views about genetic testing, highlighting benefits such as diagnostic confirmation, risk information, and better preparation for the future. Negative consequences included the burden of knowledge, moral dilemmas around reproduction, and potential impact on insurance. The offer of genetic testing was often taken up, but was felt unnecessary in some cases. Interviewees in the study reported many benefits, suggesting genetic testing should be available to this patient group. The benefits and risks identified will inform future evaluation of models of service delivery. This research was part of a wider study exploring experiences of families with retinal dystrophy.

European Journal of Human Genetics (2013) 21, 1209-1213; doi:10.1038/ejhg.2013.19; published online 13 February 2013

Keywords: retinal dystrophy; genetic testing; service delivery; qualitative interviews

\section{INTRODUCTION}

Retinal dystrophies (RD) are a diverse group of inherited conditions associated with visual loss and blindness. The group includes retinitis pigmentosa (RP), a group of clinically overlapping retinal disorders affecting between 1 in $3500-4000$ people in Europe and the USA. ${ }^{1} \mathrm{~A}$ large number of genes have been associated with RP, which can be inherited in autosomal dominant, recessive, or X-linked patterns, and with a range of other inherited retinal disorders such as cone-rod dystrophy, choroideraemia and X-linked retinoschisis. ${ }^{2}$ In some cases there are syndromic features such as deafness (Usher syndrome) or polydactyly/developmental delay (Bardet Biedl syndrome).

High genetic heterogeneity, combined with significant phenotypic overlap between related conditions, means that comprehensive clinical evaluation may be insufficient to provide definitive delineation of an underlying molecular defect, creating difficulties in providing genetic counselling. ${ }^{3,4}$ Furthermore the absence of a molecular diagnosis will preclude gene-specific therapeutic strategies. Until recently, conventional molecular diagnostics were limited to conditions such as X-linked retinitis pigmentosa (xlRP), choroideraemia and retinoschisis ${ }^{5}$ where family history or clinical examination can steer molecular testing, while array-based detection methods were implemented to identify known mutations for certain heterogeneous conditions such as Leber congenital amaurosis. ${ }^{4}$ The advent of next generation sequencing (NGS) strategies has made molecular diagnosis and genetic testing available to significantly greater numbers of families with $\mathrm{RD}^{3,6,7}$ and as new NGS tests become available, it will be important to understand where there is significant patient need for testing and to define how clinical services should meet those needs.

While the uptake, impact of and attitudes towards genetic testing have been widely reported for many inherited conditions, much less is known about its impact or clinical utility for hereditary eye disease. ${ }^{8-14}$ Some studies have demonstrated positive attitudes and support for genetic testing in $\mathrm{RD}^{15-17}$ but the potentially negative psychological consequences associated with predictive testing underline the need for counselling protocols to support families. ${ }^{15}$ A strong motivating factor to seek genetic testing for RD has been reported to be the opportunity to access novel therapies ${ }^{16}$ but, while such treatments remain unavailable for the vast majority of inherited retinal disorders, testing benefits are likely to centre on improving diagnosis, understanding of inheritance pattern and prognosis. ${ }^{17} \mathrm{RP}$ patients and at-risk family members have also reported interest in prenatal testing ${ }^{15,18,19}$ and case studies describe use of pre-natal diagnosis ${ }^{20}$ and pre-implantation genetic diagnosis. ${ }^{21}$

Evaluation of the benefits of genetic testing for RD is complex as the measurement of clinical outcomes, in the absence of clinical interventions, remains ill-defined. The aim of this study was to use qualitative methods to explore the motivations for, and impact of genetic testing amongst UK patients and their relatives. The study

${ }^{1}$ University of Manchester, Manchester, UK; ${ }^{2}$ Cardiff University Institute of Cancer \& Genetics, Cardiff, Wales, UK; ${ }^{3}$ Oxford University Hospitals NHS Trust, Oxford, UK; ${ }^{4}$ Moorfields Eye Hospital NHS Foundation Trust, London, UK; ${ }^{5}$ UCL Institute of Ophthalmology, London, UK; ${ }^{6}$ Central Manchester University Hospitals NHS Foundation Trust, Manchester, UK

*Correspondence: Georgina Hall, Genetic Medicine, St Mary's Hospital, Oxford Road, Manchester, M13 9WL, UK. Tel: +44 $1612764150 ;$ Fax +44 1612766145 ; E-mail: Georgina.Hall@cmft.nhs.uk

Received 29 October 2012; revised 6 January 2013; accepted 8 January 2013; published online 13 February 2013 
represents a first step towards understanding the clinical impact of genetic testing for RD in the UK context.

\section{METHODS}

Patients affected by a range of progressive inherited retinal disorders and their relatives were purposively sampled to identify those who (1) had a known causative genetic defect in the family, and (2) had been offered and/or made choices around genetic testing. Interviews were conducted as part of a wider study exploring service needs. Within a family, a proband was recruited who had attended an NHS multidisciplinary genetic ophthalmic clinic in one of the three centres; St Mary's Hospital (Manchester), Moorfields Eye Hospital (London), and Oxford University Hospitals (Oxford). To ensure that a diversity of opinions was represented, snowball sampling was used to recruit patients' relatives through the consenting patient interviewees. This extended participation to include people who have attended a different genetic ophthalmic clinic, or may not have attended a clinic at all. Across the three centres, interviewees came from families with a diversity of inheritance patterns and age of onset.

In-depth telephone interviews using a semi-structured questionnaire were conducted, lasting 45 to $90 \mathrm{~min}$, audio recorded and transcribed verbatim. The interviews explored interviewees' views about their clinical, family and personal experiences of genetic testing for RD. An interpretive, inductive thematic analysis was undertaken, assisted by the Atlas.ti software package. In the absence of an existing body of research literature on this topic, the thematic technique was chosen because it is flexible theoretically and analytically. ${ }^{22}$ The analysis was carried out by systematically identifying, describing, comparing/ contrasting, refining and, finally, naming and defining themes. This research was approved by NHS North West 2 Research Ethics Committee, REC reference number 10/H1005/48.

\section{RESULTS}

\section{Sample}

The sample represented a range of inheritance patterns and condition severity. It included interviewees who had been offered diagnostic, carrier and predictive testing by a genetic eye clinic (see Table 1). Forty-two patients were approached to take part in the study, twentyfour consented, and twenty were interviewed. The sample included twenty interviewees in total: nine affected individuals, ten unaffected and one suspected affected. Four of the twenty interviewees were recruited by family snowball sampling; they are denoted by an asterisk in the table.

Fourteen interviewees reported having a genetic test and five reported not having one. One individual was uncertain whether or not he had had a test. Of the five interviewees who had not had a genetic test, three women had a known carrier status and therefore did not undergo formal testing, one woman was at risk of carrying $\mathrm{X}$-linked RP, and one woman was at risk of being affected by Sorsby Fundus Dystrophy.

\section{What is the impact of genetic testing for retinal dystrophy?}

Positive attitudes towards genetic testing: information and understanding. Both affected and unaffected interviewees in this study articulated mainly positive views about genetic testing. Interviewees described valuing genetic testing because it provided information that improved the specificity of their diagnosis, which was felt to benefit the proband and their wider family (P23/affected male: 'I think if it assists in diagnosis, if it assists in pinpointing where problems lie, it can only be a good thing'). Testing was often pursued to confirm the molecular status of the affected individual (P15/likely affected female: 'I've thought, yeah, I am going to do it because I just want to know for certain, 100\% guarantee that I've got this thing.'). Another benefit was gaining a better understanding of the genetic implications of the condition.

Even where there was no overt or immediate clinical benefit- that is, the genetic test result would not lead to an alteration in clinical management-gathering information was in itself described as a strong underlying driver for wanting a genetic test (P28/affected male: 'If they could identify a particular gene mutation, they would be able to give us a bit more information about her specific condition'). Testing was also pursued out of interest (P2/unaffected female: 'There's no particular reason medically, but she wanted to have a test to find out.').

Affected and unaffected individuals also sought information about the risks of the condition to future generations. Many unaffected

Table 1 Characteristics of Patients Interviewed in this study

\begin{tabular}{|c|c|c|c|c|c|c|}
\hline P\# & Gender & Affected or Unaffected & Condition in family & Inheritance pattern & Had genetic testing? & If yes, which kind? \\
\hline 1 & M & Affected & RP & X-linked & Yes & Diagnostic \\
\hline 2 & $\mathrm{~F}$ & Unaffected & Choroideraemia & X-linked & No & $\mathrm{n} / \mathrm{a}$ \\
\hline 7 & M & Affected & $\mathrm{RP}$ & Recessive & Yes & Diagnostic \\
\hline 9 & M & Affected & $\mathrm{RP}$ & X-linked & Yes & Diagnostic \\
\hline 10 & M & Affected & $\mathrm{RP}$ & X-linked & Yes & Diagnostic \\
\hline 11 & $\mathrm{~F}$ & Unaffected & RP & X-linked & No & $\mathrm{n} / \mathrm{a}$ \\
\hline 12 & $\mathrm{~F}$ & Unaffected & $\mathrm{RP}$ & Dominant & Yes & Predictive \\
\hline 13 & $\mathrm{~F}$ & Affected & RP & Dominant & Unknown & $\mathrm{n} / \mathrm{a}$ \\
\hline 14 & M & Unaffected & Sorsby Fundus Dystrophy & Dominant & Yes & Predictive \\
\hline 15 & $\mathrm{~F}$ & Likely Affected & Sorsby Fundus Dystrophy & Dominant & No & $\mathrm{n} / \mathrm{a}$ \\
\hline 17 & $\mathrm{~F}$ & Unaffected & RP & X-linked & Yes & Carrier \\
\hline $19 *$ & $\mathrm{~F}$ & Unaffected & $\mathrm{RP}$ & X-linked & Yes & Carrier \\
\hline $20^{*}$ & $\mathrm{~F}$ & Unaffected & $\mathrm{RP}$ & X-linked & No & $\mathrm{n} / \mathrm{a}$ \\
\hline 22 & $\mathrm{~F}$ & Unaffected & Cone-rod dystrophy & Dominant & Yes & Predictive \\
\hline $23 *$ & $M$ & Affected & Cone dystrophy & Dominant & Yes & Diagnostic \\
\hline 24 & $\mathrm{~F}$ & Unaffected & Leber Congenital Amaurosis & Recessive & Yes & Carrier \\
\hline 25 & $\mathrm{~F}$ & Unaffected & XLRetinoschisis & X-linked & No & $\mathrm{n} / \mathrm{a}$ \\
\hline $26^{*}$ & M & Affected & XLRetinoschisis & X-linked & Yes & Diagnostic \\
\hline 27 & $\mathrm{~F}$ & Affected & Cone-rod dystrophy & Dominant & Yes & Diagnostic \\
\hline 28 & M & Affected & Sorsby Fundus Dystrophy & Dominant & Yes & Predictive \\
\hline
\end{tabular}


interviewees chose to be tested for carrier status (P12/unaffected female: 'I was interested to see what could be found out about the problem... and to know if I was perhaps a carrier'). By contrast, one potentially affected participant without children felt the test was unnecessary (P15/likely affected female: 'I would consider it (if I had children) because I would want to know, am I certainly passing on a genetically related disease to them?'). Some families saw genetic testing as an opportunity to make sense of the inheritance pattern and understand the context of the condition in their family (P9/affected male: 'We just wanted to check where it had come from'). A few approached testing as a family unit rather than as individuals (P13/ affected female: 'She wanted to go with her brother and her sister and all of them to be tested again').

\section{Preparation for the future}

The value of testing as a predictive tool was underlined. In cases where risk had been excluded, genetic testing can provide relief (P15/ likely affected female: 'If I had children... (and) I know he's negative now with his test. That must give some comfort'). However, even those who had a positive genetic test discussed the practical benefits of such testing in being able to adapt their lives towards the future impact of a condition. For example, a predictive test result allows the formulation of career plans and the arrangement of practical adaptations (P19/unaffected female: 'If we've got a child with bad eyesight that is going to eventually lose their eyesight, I want to know because they can be guided with career and everything else').

Interviewees acknowledged the absence of current treatments. Nonetheless, a frequently cited reason for pursuing genetic testing was to aid research, often in anticipation that treatments and cures will be developed, benefiting either themselves or future generations (P7/affected male: 'if they can identify the faulty genes, that's a good starting point to finding a cure for it').

Risks of genetic testing. Genetic testing was generally considered safe and patients regarded the risks neutrally. Several perceived there to be little or no risk in having a genetic test (P13/affected female: 'I've never thought of it as a risky thing'). Furthermore, there was trust in clinicians to provide appropriate information (P19/unaffected female: 'I think I would rely on the medical profession, that if there was any risk they would tell me'). However, psychosocial risks were discussed implicitly. Some parents discussed feelings of guilt and blame about passing on a gene. For example, a parent of an affected child discussed her mother's desire for a test because she felt guilty and wanted to accept blame for the condition (P2/Unaffected female: '[The affected child's grandmother] said that she was feeling guilty... she says, I want to take some responsibility, and I want to know').

Lack of benefits. In some instances, interviewees believed that genetic testing lacked any direct benefit. For example, one mother of an affected boy did not have the test because she showed some mild signs of the disease, essentially confirming her as a carrier (P2/unaffected female: 'I've always assumed that it's pretty clear and there's not particularly any need for a test'). Indeed, some interviewees had not been offered a test because either the diagnosis/carrier status was confirmed through clinical examination or because affection status was defined from the family history and pedigree. There were also cases where affected individuals felt that a test would not benefit them (P10/affected male: 'I'm not that particularly bothered anyway, because I've been dealt the card') or that the results of the test would not affect the treatment of the condition, rendering it inconsequential to their life (P15/likely affected female: 'I don't think the treatment would be any different even if it was discovered to be anything else').

A minority of affected interviewees in this study had not been interested in genetic testing having become aware of their genetic status through clinical investigation. One participant had a genetic test many years ago, but reported not seeking or wanting the result (P10/affected male: 'I've shunned away from [testing] anyway because I found out what I wanted to know'). This view was echoed elsewhere (participant reporting her cousin's view-P28/affected male: 'He says, 'it doesn't matter to me why I'm blind, what caused it or anything; I'm really not interested."). Importantly, two interviewees appeared not to understand why they had a genetic test (P14/unaffected male: 'I honestly couldn't pinpoint why I went for the blood test').

Negative impacts of testing. Some interviewees reported feeling that genetic testing was harmful because of the associated psychosocial risks. In other words, knowing about a negative future life change can be psychologically difficult. Some individuals articulated awareness of risks associated with pre-symptomatic testing and felt that they would rather not know what the future held (P1/affected male: Sometimes... it's better not to know, because some people can't cope with the knowing'). Similarly, a parent who was offered a test decided it was not in their or their child's best interest because of the consequences of knowing (P7/affected male: 'If you don't know, you can lead a pretty much ordinary life and be none the wiser'). Another person discussed the view of a family member who did not pursue testing (P28/affected male: 'It's not that they don't care. It's one of these things, do you want to know that something may happen to you in 25 years' time?').

The complexities of decision-making around carrier testing for parents at high risk seemed especially weighty (P17/unaffected female: 'If she has the test and is shown to be a carrier, she's got a 1 in 4 chance... to me that means that the decision she takes has a lot more weight to it'). This weight of decisions reflects the feelings of guilt or blame of passing down the gene in the family. Some interviewees also commented that genetic testing can generate further questions and prompt complex, difficult choices. For example, knowing about risk might lead to moral dilemmas such as whether or not to terminate a pregnancy (P11/unaffected female: 'It's still going to, potentially, give her a moral dilemma at some point and I think she would prefer not to have that decision to make').

Negative practical consequences were also highlighted. One potentially affected participant did not have a test because of the possible impact on her employment (P15/likely affected female: 'My concern about having a genetic test all along is what impact it would have on my work life'). A small number of affected interviewees expressed fear that having a genetic test would generate extra information, carrying a privacy risk. Concerns were expressed about the potential implications of genetic testing on insurance coverage (P12/unaffected female: 'They feel that if they have them tested and something does pop up then in the future they can't get medical insurance because it's a preexisting condition').

Nevertheless, no participant expressed regret about having a genetic test. The only retrospective negative feelings were reported by a mother who spoke about consenting to her son's diagnostic test, without fully appreciating its gravity (P2/unaffected female: 'I can't remember whether I was given a choice, because I didn't realise how significant it was'). Despite her ambivalent feelings, she later confirmed that she would not have changed her decision to consent. 
When should genetic testing be undertaken, and in what context? The amount of time it takes to decide whether or not to have the test featured strongly in the interviews. A major factor in the ambivalence for the participant above was that her son had seen several professionals in quick succession (P2/unaffected female: 'It just appeared we were moving on to a different department and we were going to have this test. And I didn't really know the score'). Conversely, in some cases several years passed before having a test (P11/unaffected female: 'She probably waited about four years' and P19/unaffected female: 'So it's only... three or four years ago that she had the test because she didn't feel it was necessary. It was something in the distance for her.'). One person said she would have a genetic test when her sight begins to degenerate (P15/likely affected female: 'For the time being I'm more than happy to take the genetic test as and when my symptoms do actually directly start affecting my eyesight but at the present time I don't intend doing it at all.').

Age was also a focus of discussion. Uncertainty was expressed about the appropriate age for an individual to understand and consent to a genetic test. One participant said that 19 years of age was too young to have a carrier test but, at 21 , one is better able to 'handle' the information (P17/unaffected female: 'Certainly at that stage I think she was too young... maybe now she is getting towards the age where she's mature enough to make that decision and able to deal with that knowledge'). Another mentioned that her family objected to her having a test at a young age (P20/unaffected female: 'I think she's just worried that I'm too young to make such decisions').

In summary, findings in this study suggest diverse attitudes towards genetic testing amongst members of retinal dystrophy families. The desire for genetic testing may vary according to personal preferences and circumstances, age and reproductive plans.

The clinical context for genetic testing. When asked to consider who should provide genetic testing services, interviewees articulated diverse views. Some interviewees believed that testing should be provided by professionals with genetics expertise, such as a clinical geneticist and/or department (P2/unaffected female: 'I felt reassured by being here in genetics' and P11/unaffected female: 'I think it should be a genetic specialist... it's their field and I think it's their specialism, so I think I would feel more confident with them'). One person proposed that testing should be provided by a team of ophthalmologists and genetic specialists (P7/affected male: 'Certainly I think it needs to be a combination of both an eye consultant and a genetic consultant'). A commonly stated view was that it would be inappropriate for non-specialists to provide the service (P2/unaffected female: 'I think the idea of it being the GP or optician-I can't imagine that they are qualified to deal with the medical and the psychological stuff'). However, a minority thought that an optician or GP could provide the service (P12/unaffected female: 'I would have thought more your doctor because your doctor knows probably more about you and your family history' and P13/affected female: 'I think initially you find out you've got something wrong... by going to your optician. So I think maybe that's where it should stem from in the first instance'). A small number believed that anyone is capable of providing testing, given appropriate counselling and post-test support (P19/unaffected female: 'I don't think it really matters. I think what really matters is... the facility after they've had the test.').

\section{DISCUSSION}

Genetic testing strategies for inherited RD are changing radically, making testing possible for the majority of affected patients and families. However, the potential benefits and harms of such testing have not been well-defined. This novel study explored the attitudes and experiences of individuals who have actually had the option of genetic testing for $\mathrm{RD}$, and identified some clear risks and benefits perceived by this group. This is one of the first studies to explore the perceived underlying need for testing and to explore whether this differs from other inherited conditions. The findings in this qualitative study may not be generalisable. Their relevance is limited by the small sample size and UK context. Rather, it is an in-depth exploration of the issues.

Interviewees expressed many positive views about genetic testing. Interestingly, they were aware of both the potential benefits and risks of such testing. Previous research has demonstrated the benefits of genetic testing for diagnosis of $\mathrm{RD}$, identifying possible opportunities for therapy and pre-natal or predictive screening. ${ }^{4}$ However, few studies have explored why RD families want genetic testing, what the perceived clinical utility of testing is, and how services should be provided to support people to make decisions about genetic testing. The key advantages of genetic testing reported in this study were the cognitive, behavioural and decisional control; ${ }^{23}$ gained by having definitive information about the condition. Interviewees wanted to know, and make sense of, the inheritance pattern in their family and risks to future generations. Defining the mode of inheritance was not raised by participants as a benefit of genetic testing which is likely to reflect the limited targeted gene testing at the time of the study for families with a clear family history or diagnosis. New NGS technology is now bringing better diagnostic testing for simplex cases and this is likely to be an important benefit for these families. Carrier testing was considered important because parents could be aware of risks and make reproductive decisions. This supports previous reports in the literature which cite 'reproductive empowerment' as a key motivating factor for carrier testing in other genetic conditions. ${ }^{24}$ Interviewees also described a need to resolve uncertainty, and reflected that predictive and diagnostic testing in particular provided information that enabled practical preparation for vision loss, for example for employment or education. Limited research around pre-natal genetic testing in retinal dystrophy suggests that the decisions about pre-natal testing and reproductive decision-making are individual. ${ }^{18}$ The ethical dilemma around reproduction was discussed by one participant, but none of the interviewees had undergone or were planning to undergo pre-natal testing.

Currently, most inherited retinal disorders are untreatable; it is not yet possible to influence the primary course of the disorder through clinical intervention. Informing future gene therapy trials has been cited as a key motivation to develop a UK molecular testing service. $^{16,17,25}$ This study's findings support this notion. Most interviewees were aware of the gene therapy's potential and valued access to genetic research because it provided hope (empowerment ${ }^{23}$ ) of gaining earlier access to novel therapies.

Despite all study interviewees being members of families with known gene mutations, gene testing was not felt necessary in all situations. Some interviewees had received sufficient clinical information on the basis of ophthalmic investigations to render testing unnecessary. Interviewees also identified some negative consequences of genetic testing, such as the burden of knowledge, moral dilemmas around decisions and the impact on insurance. All patients valued the knowledge provided by genetic testing and, while one patient described ambivalent feelings, she confirmed that she would not in retrospect have changed her decision to consent.

In keeping with other studies, genetic counselling and facilitated decision-making were found to be important elements of genetic testing services, particularly where the test may have family or 
predictive consequences. ${ }^{15,16}$ Some interviewees described confusion about the purpose of testing, possibly because it was poorly communicated by clinicians. This highlights the importance of providing complete information about the test and its uses, allowing time to decide, and fully involving patients in the decision-making process. Interviewees had mixed views about who should provide genetic testing services, but the need for accurate information and ongoing support was highlighted. Note that although the question of context was posed to interviewees generally, their views are likely to have been influenced by their experience with their specific genetic testing provider.

Inherited vision loss has been shown to impact psychological adjustment in a broad range of areas including identity, ${ }^{26}$ health-care, career, and social and family relationships. This study is the first to examine genetic testing's psychosocial impact and suggests that patients are aware of the burden of genetic knowledge. However more research is needed to understand the consequences of inherited visual loss. ${ }^{27}$

The data provide evidence in support of genetic testing provision for retinal dystrophy. Its benefits include information provision, identifying risks to self and family, aiding future preparation and decision-making, and assisting with research. The current lack of treatment for retinal dystrophy means health status outcomes are not suitable for evaluating future services. Quantitative studies will be required to explore uptake of genetic testing in families with known mutations. Also, patient-reported outcome measures such as Empowerment $^{23}$ can be used to capture data about the outcomes of genetic testing, in order to evaluate service developments in the future.

\section{CONFLICT OF INTEREST}

The authors declare no conflict of interest.

\section{ACKNOWLEDGEMENTS}

The authors would like to thank Fight for Sight who funded this research through a programme grant. The authors would also like to acknowledge the support of the Manchester Biomedical Research Centre and the Moorfields Eye Hospital Biomedical Research Centre funded by the National Institute of Health Research.

1 Berger W, Kloeckener-Gruissem B, Neidhardt J: The molecular basis of human retinal and vitreoretinal diseases. Prog Retin Eye Res 2010; 29: 335-375.

2 Daiger SP, Bowne SJ, Sullivan LS: Perspective on genes and mutations causing Retinitis pigmentosa. Arch Ophth 2007; 125: 151-158.

3 O'Sullivan J, Mullaney BG, Bhaskar SS et al: A paradigm shift in the delivery of services for diagnosis of inherited retinal disease. J Med Genet 2012; 49: 322-326.
4 Koenekoop RK, Lopez I, den Hollander Al, Allikmets R, Cremers FP: Genetic testing for retinal dystrophies and dysfunctions: benefits, dilemmas and solutions. Clin Experiment Ophthalmol 2007; 35: 473-485.

5 Henderson RH, Waseem N, Searle R et al: An assessment of the apex microarray technology in genotyping patients with Leber congenital amaurosis and early-onset severe Retinal dystrophy. Invest Ophthalmol Vis Sci 2007; 48: 5684-5689.

6 Neveling K, Collin RWJ, Gilissen C, van Huet RAC et al: Next-generation genetic testing for retinitis pigmentosa. Hum Mutat. 2012; 33: 963-972.

7 Shanks ME, Downes SM, Copley RR et al: Next-generation sequencing (NGS) as a diagnostic tool for retinal degeneration reveals a much higher detection rate in earlyonset disease. Eur J Hum Genet 2012; e-pub ahead of print 12 September 2012; doi:10.1038/ejhg.2012.172.

8 Cameron LD, Muller C: Psychosocial aspects of genetic testing. Curr Opin Psychiatr 2009; 22: 218-223.

9 Marteau TM, Coyle RT: Psychological responses to genetic testing. BMJ 1998; 316 : 693-696.

10 Broadstock M, Michie S, Marteau T: Psychological consequences of predictive testing: a systematic review. Eur J Hum Genet 2000; 8: 731-738.

11 Meiser M, Dunn S: Psychological impact of genetic testing for Huntington's disease: an update of the literature. J Neurol Neurosurg Psychiatry 2000; 68: 574-578.

12 Watson M, Foster C, Eeles R et al: Psychosocial impact of Breast/Ovarian (BRCA1/2) cancer predictive testing in a UK multi-centre clinical cohort. Br Journal Cancer 2004; 91: 1787-1794.

13 Dancyger C, Smith JA, Jacobs C, Wallace M, Michie S: Comparing family members' motivations and attitudes towards genetic testing for hereditary Breast and Ovarian cancer: a qualitative analysis. Eur J Hum Genet 2010; 18: 1289-1295.

14 Burton H, Alberg C, Moore AT: Genetics in ophthalmology: equity in service provision? J Public Health-UK 2010; 32: 259-266.

15 Mezer E, Babul-Hirji R, Wise R et al: Attitudes regarding predictive testing for Retinitis pigmentosa. Ophthalmic Genet 2007; 28: 9-15.

16 Bong C, Potrata B, Hewison J, McKibbin M: Attitudes of patients and relatives/carers towards genetic testing for inherited retinal disease. Eye (Lond) 2010; 24: $1622-1625$.

17 Moore T, Burton $\mathrm{H}$ : Genetic ophthalmology in focus: A needs assessment and review of specialist services for genetic eye disorders. PHG Foundation 2008; http:// www.phgfoundation.org/pages/work2.htm. pp 29-39.

18 Furu T, Kääriäinen H, Sankila EM, Norio R: Attitudes towards prenatal diagnosis and selective abortion among patients with Retinitis pigmentosa or Choroideraemia as well as among their relatives. Clin Genet 1993; 43: 160-165.

19 Pawlowitzki IH, Rüther K, Brunsmann F, von Gizycki R: Acceptability of prenatal diagnosis for Retinitis pigmentosa. Lancet 1986; 2: 1394-1395.

20 Schwartz M, Rosenberg T: Prenatal diagnosis of Choroideraemia. Acta Ophthalmol Scand Suppl 1996; 219: 33-36.

21 Sohrab MA, Allikmets R, Guarnaccia MM, Smith RT: Preimplantation genetic diagnosis for Stargardt disease. Am J Ophthalmol 2010; 149: 651-655.

22 Braun V, Clarke V: Using thematic analysis in psychology. Qual Res Psychol 2006; 3: 77-101.

23 McAllister M, Dunn G, Chris T: Empowerment: qualitative underpinning of a new clinical genetics-specific patient-reported outcome. Eur J Hum Genet 2011; 19: $125-130$.

24 Lewis C, Skirton H, Jones R: Reproductive empowerment: The main motivator and outcome of carrier testing. J Health Psychol 2011; 17: 567-578.

25 Lorenz B, Preising M, Stieger K: Retinal blinding disorders and gene therapymolecular and clinical aspects. Curr Gene Ther 2010; 10: 350-370.

26 Hayeems RZ, Geller G, Finkelstein D, Faden RR: How patients experience progressive loss of visual function: a model of adjustment using qualitative methods. $\mathrm{Br} J$ Ophthalmol 2005; 89: 615-620.

27 Jangra D, Ganesh A, Thackray R et al: Psychosocial adjustment to visual loss in patients with Retinitis pigmentosa. Ophthalmic Genet 2007; 28: 25-30. 